\title{
Scientific Uncertainty and Climate Change: Part I. Uncertainty and Unabated Emissions
}

\author{
Stephan Lewandowsky • James S. Risbey • \\ Michael Smithson • Ben R. Newell • John \\ Hunter
}

November, 2013

\begin{abstract}
Uncertainty forms an integral part of climate science, and it is often used to argue against mitigative action. This article presents an analysis of uncertainty in climate sensitivity that is robust to a range of assumptions. We show that increasing uncertainty is necessarily associated with greater expected damages from warming, provided the function relating warming to damages is convex. This constraint is unaffected by subjective or cultural risk-perception factors, it is unlikely to be overcome by the discount rate, and it is independent of the presumed magnitude of climate sensitivity. The analysis also extends to "second-order" uncertainty; that is, situations in which experts disagree. Greater disagreement among experts increases the likelihood that the risk of exceeding a global temperature threshold is greater. Likewise, increasing uncertainty requires increasingly greater protective measures against sea level rise. This constraint derives directly from the statistical properties of extreme values. We conclude that any appeal to uncertainty compels a stronger, rather than weaker, concern about unabated warming than in the absence of uncertainty.
\end{abstract}

Keywords Uncertainty · unabated emissions · decision making

\section{Introduction}

Uncertainty is an unavoidable aspect of scientific endeavors. The IPCC's Fourth Assessment Report of 2007 used the word "uncertain" or its derivatives more than 1200 times in the report of Working Group 1 alone - or around 1.2 times per printed page. Although the relevant scientific community has sought to develop ways of dealing with uncertainty (e.g., Intergovernmental Panel on Climate Change, 2005; Narita, 2012), scientific uncertainty has often been highlighted in public debates to preclude or delay political action on a contentious issue (e.g., Freudenburg, Gramling, \& Davidson, 2008; Freudenburg \& Muselli, 2013).

University of Western Australia and University of Bristol E-mail: stephan.lewandowsky@bristol.ac.uk · CSIRO Marine and Atmospheric Research, Hobart, Tasmania - Australian National University - University of New South Wales · Antarctic Climate \& Ecosystems Cooperative Research Centre, Hobart, Tasmania 


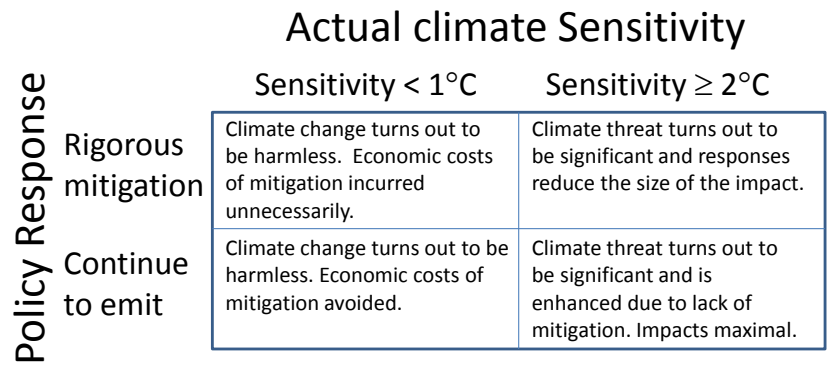

Fig. 1 Contingency table relating the (in principle unknowable) true state of the world (columns) to possible policy decisions (rows). Cell entries are the consequences that are likely to arise from each policy decision contingent on the two possible states of the world. For simplicity, the range of possible equilibrium climate sensitivities (i.e., the ultimate response of the climate system to a doubling in $\mathrm{CO}_{2}$ levels from preindustrial times) is dichotomized into values below $1^{\circ} \mathrm{C}$, which would entail only limited adverse consequences, and values in the vicinity or above $2^{\circ} \mathrm{C}$, which would lead to serious adverse consequences on an unabated emissions path.

Politically motivated appeals to uncertainty tend to follow two streams of argumentation: The first stream asymmetrically focuses on the possibility that the problem (e.g., climate change) may be less serious than anticipated and that any potential surprises are more likely to be benign rather than inauspicious. The second stream paints scientific uncertainty as a "monster" that is not readily managed (Van der Sluijs, 2005). Accordingly, some have argued that science makes environmental controversies worse (Sarewitz, 2004), implying that research is impotent in informing public policy.

We argue that those streams of argumentation misconstrue the impact of scientific uncertainty on potential policy choices. In the remainder of this article we show that, in the case of climate change, greater uncertainty about the evolution of the climate implies a greater probability of adverse consequences. In a companion article, we analyze the implications of uncertainty on mitigation efforts (Lewandowsky, Risbey, Smithson, \& Newell, in press) and show similarly that greater uncertainty implies a greater, not lesser, impetus for mitigative action.

\section{Framing Uncertainty: Risks and Outcomes}

We begin by highlighting the interaction between policy decisions and the true state of the world. Figure 1 presents two possible global "policy actions" (in rows) and two possible states of the world (in columns), with the possible outcomes highlighted in the corresponding cells.

By dichotomizing a range of possible decisions and states of the world, the figure necessarily simplifies. Nonetheless, it highlights several core issues. First, it emphasizes a fact that receives insufficient attention for historical and psychological reasons (Lewandowsky, Oreskes, Newell, Smithson, \& Risbey, 2013); namely, that the decision not to cut emissions is not an inactive "null" default. Instead, withholding emission cuts and continuing with "business as usual" equates to an active decision to add greenhouse gases to the atmosphere. This realization is 
non-trivial because it reframes the issue from whether or not societies "should do something," to an acknowledgment that we are doing something already-namely, adding $\mathrm{CO}_{2}$ to the atmosphere - and that the consequences of that action must be contrasted with the costs of alternative actions.

This raises the second point made by Figure 1, that the outcomes of actions must be evaluated with respect to the true states of the world. There are two main approaches by which the constraints implied by Figure 1-and the associated uncertainties - are conventionally resolved: One approach relies on economic costbenefit analysis and the other relies on the precautionary principle.

\subsection{Cost-benefit analysis}

Cost-benefit analysis (CBA) considers the cost of various emission paths against the associated benefits accrued by a reduction or avoidance of damages from climate change (e.g., Garnaut, 2011; Nordhaus, 2010; Stern, 2007; Tol, 2011). Uncertainty can be represented in CBA by probability-weighting the competing costs (cf. Schneider, 2002).

Several limitations curtail the utility of CBA: First, notwithstanding its apparent objectivity, CBA is not free of ethical considerations (Aldred, 2009; Nolt, 2011; Risbey, 2006), even if they are tacit and remain unspecified. This problem is most apparent in connection with the monetarization of environmental "goods" such as species diversity (Ressurreição et al., 2011), the existence of songbirds (Funtowicz \& Ravetz, 1994), or indeed human life (cf. Li, Vietri, Galvani, \& Chapman, 2010). Second, CBA is affected by people's attitudes toward risk (Nordhaus, 2011) and is therefore necessarily influenced by cultural and political factors (e.g., Kahan, Slovic, Braman, \& Gastil, 2006; Slovic, 1999). To illustrate, Ackerman, Heinzerling, and Massey (2005) showed retrospectively that CBA would have prevented a number of regulatory public-health measures from being implemented, among them limiting the exposure to vinyl chloride in the workplace which likely saved many lives (Michaels, 2008).

Third, because climate mitigation costs would be incurred now, whereas the anticipated damages from climate change will largely come due in the future, CBA must necessarily discount those future costs against present expenditure by applying an interest rate (the discount rate; e.g., Anthoff, Tol, \& Yohe, 2009). Decisions about the discount rate have dramatic effects on the outcome of CBA; if the discount rate is sufficiently high, any future cost, no matter how great, will appear minuscule compared to present-day mitigation costs (e.g., Weitzman, 2010a). For example, with a discount rate of $1 \%$ per annum, a presumed damage of $\$ 1,000,000$ in 300 years is worth around $\$ 50,000$ today. With a rate of $5 \%$, the discounted value is less than 50 cents (Sterner \& Persson, 2008). Relatively small variations in discounting can thus alter the anticipated damage cost by orders of magnitude - thereby undermining the robustness of CBA.

\subsection{Precautionary principle}

An alternative to CBA invokes the precautionary principle (e.g., Vlek, 2010a, 2010b). Put in its most succinct form, the precautionary principle holds that if 
there is a potential for harm from an activity, and if there is uncertainty about the magnitude of those impacts, then action should be taken to avoid that harm (e.g., Gardiner, 2006). Unlike CBA, the precautionary principle is asymmetrical because it focuses on the bottom-right cell in Figure 1, thereby effectively reversing the "burden of proof": Unless an activity (or product) can be shown to have no harmful consequences, it should cease. Thus, on the precautionary principle, action is triggered because there is uncertainty about an outcome, not despite of any uncertainty.

Like CBA, the precautionary principle has been subject to extensive criticism (e.g., Feintuck, 2005; John, 2010; Kahan et al., 2006; Peterson, 2006; Vlek, 2010a, 2010b). For example, the principle has been labeled incoherent (Peterson, 2006) because if any precautionary action (e.g., cutting $\mathrm{CO}_{2}$ emissions) itself leads to adverse outcomes (e.g., risks from an expansion of nuclear power), then the precautionary principle provides no guidance about how to resolve the conundrum. Further criticism has focused on the ill-specified role of uncertainty, which by an extreme interpretation might suggest that an activity should be banned if there is any possibility, no matter how small, that it might prove harmful (cf. Gardiner, 2006).

We therefore suggest that neither CBA nor the precautionary principle are sufficient to resolve the dilemma posed by Figure 1. We argue instead that the implications of Figure 1 are best explored by deriving uncertainty-based constraints that are (a) not subject to cultural and personal vagaries of risk perception, (b) are unlikely to be affected by the discount rate, and (c) involve few if any ethical considerations. Our approach rests on developing ordinal constraints - i.e., constraints of the form "greater than" or "lesser than" - that derive from the functional form of the mapping between uncertainty and outcomes.

\section{Uncertainty and Unabated Emissions}

In the remainder of this article, we consider the role of uncertainty with respect to the policy decision to continue with unabated emissions (bottom row of Figure 1). The complementary role of uncertainty with respect to mitigation is addressed in a companion article (Lewandowsky et al., in press). The companion article additionally shows how such uncertainty-based constraints might be amenable to informing policy choices via a simple decision model, such as a "safe-minimumstandard" approach (i.e., specifying the maximum tolerable risk of exceeding a threshold such as an increase in global temperatures of $2^{\circ} \mathrm{C}$ ).

We differentiate and delineate the concepts of risk and various types of uncertainty as follows. We use risk to refer to the set of possible consequences of climate change, each with quantifiable probabilities and quantifiable losses (Schneider, 2002). We use uncertainty, by contrast, to refer to the imprecision of our knowledge of various crucial climate variables, which is typically captured by the variance or standard deviation of the variable's estimate (cf. Padilla, Vallis, \& Rowley, 2011). For the most part, we will be concerned with uncertainty in equilibrium climate sensitivity (ECS); that is, uncertainty about the warming ultimately expected in response to a doubling of $\mathrm{CO}_{2}$ from preindustrial times. For convenience, we assume that $\mathrm{CO}_{2}$ will double, from approximately $275 \mathrm{ppm}$ pre-industrially to 550 
ppm, such that the expected mean global temperature increase equals the presumed sensitivity.

The value of climate sensitivity is constrained by several sources of evidence, ranging from paleoclimatology (e.g., Hegerl, Crowley, Hyde, \& Frame, 2006; Zeebe, Zachos, \& Dickens, 2009) to analysis of the modern observational temperature record in conjunction with climate modeling (e.g., Bender, Ekman, \& Rodhe, 2010). Those multiple lines of evidence converge on a point estimate of equilibrium climate sensitivity of around $3{ }^{\circ} \mathrm{C}$, with a likely range from $2{ }^{\circ} \mathrm{C}$ to $4.5^{\circ} \mathrm{C}$, and a lower bound of $1.5^{\circ} \mathrm{C}$ (e.g., Knutti \& Hegerl, 2008; Meehl et al., 2007). Values substantially higher than $4.5^{\circ} \mathrm{C}$ remain subject to debate, with some arguing that it ought to be considered as a firm upper bound (Annan \& Hargreaves, 2011) and others suggesting the contrary (Roe \& Armour, 2011).

In light of those multiple converging constraints, we consider it appropriate to commence our analysis by focusing on "first-order" uncertainty (cf. Walley, 1991); that is, the unavoidable error surrounding the (single) estimate of a parameter that is captured by the variance of its inferred probability density function. To prevent confusion, we call this quantity uncertaintyecs (for Equilibrium Climate Sensitivity) from here on, and we will introduce other subscripts to differentiate it from other manifestations of uncertainty where necessary. We later extend our analysis to "second-order" uncertainty; where the nature of the probability distribution is uncertain, so that its variance and higher moments are unknown. Second-order uncertainty may arise from sheer lack of relevant information, but also when there are divergent estimates of a parameter such as climate sensitivity (cf. Smithson, 1999).

\subsection{Asymmetrical tails}

A crucial feature of extant climate sensitivity distributions is their asymmetry: they are thought to have a "fat" upper tail and a comparatively abruptly truncated lower tail. That is, the area of the distribution below its mean is more sharply bounded (i.e., it covers a narrower range) than the area above: values of climate sensitivity far above the central location of the distribution are more likely than values far below (Roe \& Baker, 2007). The fat upper tail implies that particularly severe consequences arising from continued $\mathrm{CO}_{2}$ emissions cannot be ruled out (Weitzman, 2011). This concern is supported by episodes of rapid climate change in the geological past (e.g., Bahn, Edwards, Knutti, \& Stocker, 2011; Holmes, Lowe, Wolff, \& Srokosz, 2011).

A further consideration concerns the magnitude of uncertaintyecs. To examine the impact of the magnitude of uncertainty ${ }_{\mathrm{ECS}}$, its effects must be disentangled from the value of the "best estimate" for climate sensitivity. This can be accomplished in several ways, depending on one's choice of "best estimate", or measure of central location of the sensitivity distribution. Figure 2 illustrates two possible approaches, one using the mode (panel A) and the other using the mean (panel B), as the measure of location that is to be disentangled from the distribution's spread (i.e., standard deviation). Each panel shows three arbitrary but fat-tailed probability density functions of differing standard deviations. In the top panel (A), the mode (labeled $M o$ ) is kept constant as the standard deviation $(\sigma)$ increases. This approach might appear appealing because it creates an increasingly fatter 
tail while keeping the most likely estimate unchanged. However, one consequence of keeping the mode constant while increasing spread is that the mean (vertical lines labeled $\mu$ in the figure) increases together with the standard deviation. It follows that the consequences of increasing uncertainty $(\sigma)$ cannot be disentangled from the contribution of the increasing mean, thereby preventing an unambiguous interpretation. Note also that the median likewise increases with increasing uncertainty.

This problem can be circumvented by keeping the mean of the distributions constant while increasing its spread (panel B in Figure 2). This approach is known as a mean-preserving examination of uncertainty and is common in economics and finance (e.g., Hartman, 1972). The approach is conservative because as uncertainty increases with the mean constant, the mode of the distribution (like its median) decreases; hence the effects of increasing uncertainty cannot be attributed to a concomitant increase in any measure of central location. We therefore apply the mean-preserving approach throughout.

We demonstrate the effects of a mean-preserving increase in uncertainty using a simulated lognormal distribution of climate sensitivities. The lognormal was chosen for illustrative purposes because it has a fat upper tail, but this choice entails no commitment to the precise shape of the sensitivity distribution. Table 1 summarizes the parameter values and results based on 10,000 simulated samples. The expected value of the distribution, $\mu_{L}$, was kept constant in all simulation runs, but the uncertainty of its estimate - that is, the standard deviation of the distribution; $\sigma_{L}$-increased from small to considerable across simulation runs.

Table 1 illustrates two principal consequences of increasing uncertaintyecs. First, as the spread of the distribution increases, the probability of a gravely concerning outcome also increases (columns $P\left(X>T_{c}\right)$; i.e., warming in excess of $5^{\circ} \mathrm{C}$ or even $7^{\circ} \mathrm{C}$; Sherwood \& Huber, 2010). For a threshold temperature of $5^{\circ} \mathrm{C}$, increasing uncertaintyECS fivefold, from .5 to 2.5 , increases the likelihood of catastrophe by a factor of nearly 250 , from $.06 \%$ to $14.5 \%$.

This is a necessary consequence of the fact that if two lognormal distributions have an identical expected value $\left(\mu_{L}\right)$, then the distribution with the greater standard deviation (call that $\sigma_{L 2}$ ) will always have a "fatter tail" than the distribution with the lesser standard deviation $\left(\sigma_{L 1}\right)$. Intuitively, this phenomenon arises because the lower bound of all lognormal distributions is zero, and a greater standard deviation must therefore translate into greater probability mass somewhere in the upper tail. Formally, there exists a threshold $\theta$, such that for any $x>\theta,\left\{1-c d f_{\sigma_{L 2}}(x)\right\}>\left\{1-c d f_{\sigma_{L 1}}(x)\right\}$, where $c d f$ is the lognormal cumulative density function. The precise location of $\theta$ depends on both $\mu_{L}$ and $\sigma_{L}$; here, we are concerned only with the fact that there exists a $\theta$ beyond which the distribution with the greater standard deviation has more probability mass, not the location of that threshold. We conclude that the greater the uncertainty, the greater the potential for large changes because greater uncertaintyeCs necessarily entails a greater likelihood of extremely high values of sensitivity. Rive and Myhre (2012) have recently drawn attention to the need to communicate the possibility of such extreme temperature changes to the public and policy makers.

The second principal feature of Table 1 (columns $P\left(X<T_{c}\right)$ ) appears comforting at first glance: As uncertainty ${ }_{\mathrm{ECS}}$ increases, the proportion of lower-end sensitivities increases. This is a necessary consequence of keeping the mean, $\mu_{L}$, constant while the mode and median shift to the left as uncertainty increases (cf. 



Fig. 2 The effects of increasing the spread (standard deviation, $\sigma$ ) on an arbitrary but fattailed probability density function obtained by convolving a Gaussian and an exponential distribution. (The convolution of those two distributions, defined by three parameters, lends itself particularly well to manipulation of mean, standard deviation, and "fatness" of the tail.) Panel A shows the effects of keeping the mode $(M o)$ constant at 1.4 while increasing the distribution's standard deviation, $\sigma$, from .54 to 1.14 and 1.36 , respectively. The accompanying increase in means is denoted by $\mu_{1}$ through $\mu_{3}$. Panel $\mathrm{B}$ shows the effects of keeping the mean $(\mu)$ constant at 3.2 while increasing $\sigma$ from .52 to 1.0 and 1.24 , respectively. Mo $\mathrm{Mhrough}^{\mathrm{Mo}_{3}}$ denote the accompanying decrease in modes.

Figure 2B). The proportion of low estimates $\left(<2^{\circ} \mathrm{C}\right)$ increases to $42.5 \%$ when uncertainty ${ }_{\mathrm{ECS}}$ is greatest. The large proportion of low sensitivities invites a potential alternative interpretation: With a nearly even chance that sensitivity might fall below the ostensibly-safe "guardrail" of $2^{\circ} \mathrm{C}$ warming, perhaps one could legitimately ignore the upper tail, however fat it might get with increasing uncertainty? Further analysis reveals this gamble to be inadvisable for reasons involving 
Table 1 Parameter values and results for our simulations involving a lognormal climate sensitivity distribution.

\begin{tabular}{crrrrrrrr}
\hline & \multicolumn{2}{c}{ Lognormal } & \multicolumn{2}{c}{ Gaussian $^{a}$} & \multicolumn{2}{c}{$P\left(X>T_{c}\right)^{b}$} & \multicolumn{2}{c}{$P\left(X<T_{c}\right)^{c}$} \\
Simulation & $\mu_{L}$ & $\sigma_{L}$ & $\mu_{G}$ & $\sigma_{G}$ & $5^{\circ} \mathrm{C}$ & $7^{\circ} \mathrm{C}$ & $\mu_{L}\left(3^{\circ} \mathrm{C}\right)$ & $2^{\circ} \mathrm{C}($ "safe" $)$ \\
\hline $\mathrm{A}$ & 3.00 & 0.5 & 1.08 & 0.17 & .0006 & .0 & .536 & .009 \\
$\mathrm{~B}$ & 3.00 & 1.0 & 1.05 & 0.32 & .044 & .003 & .568 & .044 \\
$\mathrm{C}$ & 3.00 & 1.5 & 0.99 & 0.47 & .101 & .020 & .595 & .267 \\
$\mathrm{D}$ & 3.00 & 2.5 & 0.83 & 0.73 & .145 & .068 & .635 & .425 \\
\hline
\end{tabular}

${ }^{a}$ Lognormal distributions are often characterized by the parameters of the underlying Gaussian distribution, shown here as $\mu_{G}$ and $\sigma_{G}$. They are related to the parameters of the lognormal distribution via $\mu_{L}=\exp \left(\mu_{G}+.5 \sigma_{G}^{2}\right)$ and $\sigma_{L}=\exp \left(\mu_{G}+.5 \sigma_{G}^{2}\right) \times \sqrt{\exp \left(\sigma_{G}^{2}\right)-1}$.

$b$ Proportions of sampled climate sensitivities that exceed the given threshold temperature $T_{c}$.

${ }^{c}$ Proportion of sampled climate sensitivities below the given threshold temperature $T_{c}$

the functional form of the relationship between continued warming and damage costs.

\subsection{The convex damage function}

There is pervasive agreement among economic models that further global warming will incur costly damage (e.g., Nordhaus, 2010; Tol, 2011). There is also agreement that the function that relates warming to cost is convex - that is, damage costs are accelerating with increasing warming. For example, one parameterization of the damage function favored by Nordhaus is:

$$
d(t)=-0.0045 \times T(t)+0.0035 \times T^{2}(t),
$$

where $d(t)$ is damage cost, expressed as a percentage of world GDP, at time $t$ as a function of temperature increase $\left(T\right.$; in ${ }^{\circ} \mathrm{C}$ ) since pre-industrial times (for a discussion of various damage functions, see Wouter Botzen \& van den Bergh, 2012; Tomassini et al., 2010; Weitzman, 2010b).

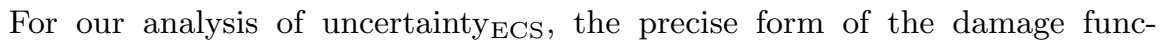
tion turns out to be of no consequence: What matters instead are three pervasive attributes of damage functions: First, beyond a minimum at quite low temperature increases, damage functions are monotonically increasing with further global warming. For example, the function in Equation 1 reaches its minimum at $T=.64^{\circ} \mathrm{C}$. Given that temperatures have already increased approximately $0.8^{\circ} \mathrm{C}$ compared to pre-industrial times, further warming from here on - or from a nearby temperature threshold (Tol, 2009) — will incur increasingly greater damages. Second, a pervasive attribute of damage functions is that they are assumed to be convex - i.e., they are upward accelerating. Third, although a damage function cannot be computed without choosing a discount rate, the function retains its 
convex shape irrespective of the value of the discount rate (e.g., Tomassini et al., 2010, Figure 5a). This convexity turns out to have notable implications.

With a convex function, we can rely on a theorem known as Jensen's inequality (Jensen, 1906) to derive ordinal predictions about the damage expected from climate change. Jensen's inequality can be stated as: "If $X$ is a (non-degenerate) random variable taking values in an interval $(r, s)$, and if $u(X)$ is a strictly convex function on $(r, s)$, then mean $[u(X)]>u($ mean $[X])$, providing that mean $[X]$ and mean $[u(X)]$ exist and are finite" (Brewster, Graham, \& Mutch, 2005, p. 394). We explore the implications of Jensen's inequality, namely that increasing variance in $X$ elevates the response mean $(u[X])$ if $u$ is convex (Smallwood, 1996), by Monte Carlo simulation.

Figure 3 shows the relationship between simulated lognormal distributions of climate sensitivity and the total risk from climate change - that is, the distribution of likely damage costs. This illustrative simulation used a simple quadratic cost function, $d(T)=T^{2}$, where $T$ was the value drawn from the climate-sensitivity distribution (bottom horizontal graphs in each panel) and $d$ was the corresponding "reflected" observation in the damage-cost distribution (vertical graphs on left). The damage function is shown in the large plot within each panel.

We again fixed the mean of the sensitivity distributions for the reasons noted earlier, and changed only uncertainty $y_{\mathrm{ECS}}$. The figure reveals that increased uncertainty $\mathrm{ECS}_{-}$ reflected in the increased spread of the climate sensitivity distribution in panel $\mathrm{B}$ compared to panel A - translates into greater expected cost: The mean of the damage cost distribution is greater in panel $\mathrm{B}$ than in panel A (compare the length of vertical double-headed arrows between panels). This result is a necessary outcome provided the function relating temperature increases (i.e., sensitivity) to cost is convex (e.g., Smallwood, 1996) and it holds - by Jensen's inequality-irrespective

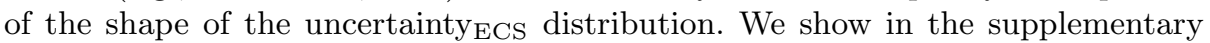
material (Section S1) that this conclusion also holds when risk is measured in ways other than mean expected damage; for example, by examining the consequences for certain thresholds of sensitivity.

A few additional points apply to Figure 3: First, not only does greater uncertaintyeCS increase the mean expected damage cost, but it also increases the uncertainty around that expected damage (call that uncertainty $y_{D}$ ). The increase in uncertainty is relevant because it must be taken into account when assessing the total risk from climate change. Second, we show in the supplementary material that this result holds irrespective of the absolute value of mean sensitivity. Although increasing sensitivity increases the expected mean damage, there always is an additional contribution from an increase in uncertaintyecs (see Table S1).

\subsection{Speed of warming and the discount rate}

Finally, we examine the relationship between climate sensitivity and the rate of future warming. There is considerable evidence that greater sensitivity translates into more rapid warming, all other things being equal (e.g., Bahn et al., 2011; Raupach et al., 2011; Ross, Matthews, Schmittner, \& Kothavala, 2012; Winton et al., 2013). It follows that greater uncertainty about climate sensitivity translates not only into greater expected damage, but it also implies that greater damages 



Climate sensitivity $(\mathrm{C})$

B

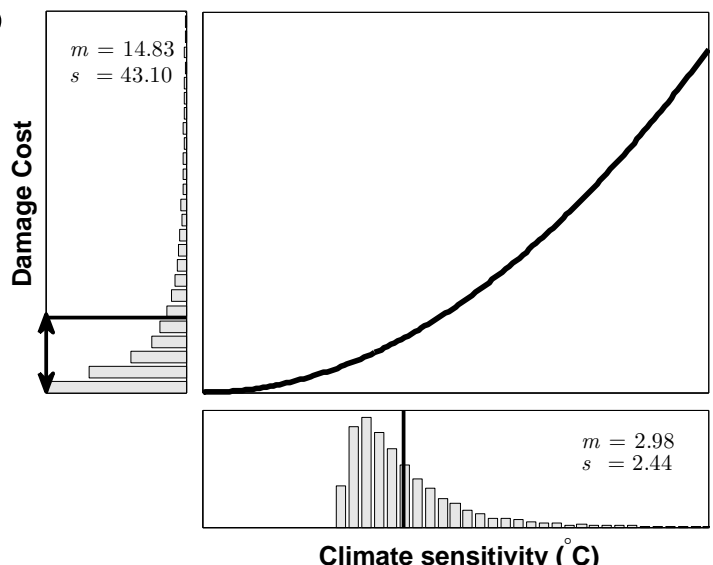

Fig. 3 Illustration of the consequences of Jensen's inequality across two levels of uncertainty $y_{\mathrm{ECS}}$. Standard deviations of the lognormal climate sensitivity distributions $\left(\sigma_{L}\right)$, shown at the bottom of each panel, are .5 and 2.5 in panels $\mathrm{A}$ and $\mathrm{B}$, respectively, with $\mu_{L}$ fixed at 3. Distributions of expected damage costs are shown at the left in each panel, using units that are arbitrary but with a scale that is aligned between panels. Mean $(m)$ and standard deviation $(s)$ of the simulated distributions are also shown. For all distributions the means are represented by thick solid lines. Vertical arrows highlight distance of the mean of the damage distribution from 0 . The damage function in the large plot within each panel is arbitrary and shown for illustration only.

are likely to arrive sooner rather than later. ${ }^{1}$ We provide a more formal analysis of the relationship between speed of warming and climate sensitivity in the supplementary material (Section S2).

This point has an important implication because it prevents the discount rate from altering the functional relationship between uncertainty $\mathrm{ECS}_{\mathrm{S}}$ and the mag-

1 Notwithstanding the greater speed of warming, the climate system will take longer overall to reach equilibrium if climate sensitivity is greater (Hansen et al., 1985). 
nitude of damages. To clarify why that is the case, it is helpful to consider the consequences of the opposite outcome, viz. if greater damages were delayed, rather than accelerated, as a function of greater uncertaintyECS. If that opposite outcome were the case, then an expected damage cost of, say, $\$ 100,000,000$ under greater uncertainty ${ }_{\mathrm{ECS}}$ would occur some time later than a cost of, say, $\$ 80,000,000$ if uncertainty ${ }_{\mathrm{ECS}}$ were lower. When a greater cost is delayed relative to a lesser one, its value can always be discounted below the lesser cost by choosing a convenient discount rate. We noted earlier that the choice of discount rate can alter discounted amounts by several orders of magnitude: For our analysis it is therefore important that greater uncertaintyECS translates into acceleration as well as amplification of climate-related damages. Because discounting, by definition, only affects future outcomes, any cost that occurs sooner than another one cannot be discounted below the other one: $\$ 100,000,000$ due now cannot be discounted to be less than $\$ 80,000,000$ that is due at some later time. This argument is again ordinal in nature and does not depend on how much quicker warming is if climate sensitivity is greater than anticipated: Provided greater sensitivity does not delay a given extent of warming - and it does not (Bahn et al., 2011; Raupach et al., 2011; Ross et al., 2012; Winton et al., 2013) - the relationship between uncertaintyECS and damages just uncovered holds irrespective of the discount rate.

Our analysis thus far rested on three assumptions. First, we assumed an asymmetric "fat-tailed" distribution of climate sensitivities. Second, by focusing on a mean-invariant transformation of a presumed probability density function of climate sensitivity, our analysis has been limited to what is often called "first-order" uncertainty; namely, uncertainty associated with the estimate of a single parameter. Third, we assumed that the function relating warming to damages is convex, as is pervasively assumed in economic modeling. We now show that our analysis remains unaffected by a relaxation of those assumptions.

\subsection{Symmetrical climate sensitivity}

We show in the supplementary material (Section S3) that the implications of Jensen's inequality generalize to situations in which the distribution of climate sensitivities is entirely symmetrical. Although the assumption of symmetry is likely incorrect (Roe \& Baker, 2007, 2011), the removal of a fat upper tails is also the most conservative assumption one can make about the evolution of the climate. The fact that increasing uncertainty nonetheless leads to increasing expected damages attests to the robustness of our approach.

\subsection{Ambiguity among estimates of climate sensitivity}

We argued earlier that enough is known about climate sensitivity to consider its estimation to involve mainly "first-order" uncertainty, or uncertainty intrinsic to the estimate of a single value. What are the implications of introducing "secondorder" uncertainty, for example by considering competing and divergent estimates of climate sensitivity?

Figure 4 (Panel A) illustrates second-order uncertainty with 3 hypothetical climate-sensitivity distributions that differ in mean and variance. Each distribution 
can be taken to represent the knowledge of a single expert obtained by an expertelicitation methodology (e.g., Morgan \& Keith, 1995). Although the hypothetical distributions share a common (lognormal) shape and overlap considerably, there is also some heterogeneity among the expert judgments. This is best illustrated by considering the areas of the curves that exceed a cutoff $\left(T_{c}\right)$, which in Figure 4 was arbitrarily set to $4^{\circ} \mathrm{C}$. Note that the same cutoff applies to all three distributions, and that the area exceeding the cutoff is a function of both mean and variance of each distribution.

Given that we assume a doubling of atmospheric $\mathrm{CO}_{2}$ throughout this article, the shaded areas also represent the probabilities $\pi_{i}$ that each expert $i$ assigns to the risk that global temperatures may increase by more than $T_{c}\left(4^{\circ} \mathrm{C}\right)$. Second-order uncertainty, then, is captured by the distribution of those exceedance probabilities, $\pi_{i}$ 's, across experts. Panel B in Figure 4 shows a hypothetical Beta distribution that characterizes those exceedance probabilities across a presumed population of experts. (Panel B also shows the three exceedance probabilities from Panel A for illustrative purposes.)

It is intuitively obvious that as the disagreement among experts increases thereby introducing greater heterogeneity into their estimated exceedance probabilitiesthe variance of the Beta distribution that characterizes those probabilities also increases. This in turn necessarily implies that there is greater probability mass over the larger values of $\pi_{i}$, thereby raising the likelihood of greater risks of exceeding $T_{c}\left(4^{\circ} \mathrm{C}\right)$.

This intuition is formalized in Figure 5, which plots a family of complementary cumulative distribution functions (i.e., the tail distribution $\bar{F}(x)=\mathrm{P}(X>$ $x)=1-F(x))$ for Beta distributions of constant mean $\left(\mu_{B}=0.2\right)$ but differing variance. The weight of the lines in Figure 5 maps into the variance of the underlying distribution: It is clear that as the variance increases, the probability mass over the larger exceedance probabilities increases. Conceptually, this means that as disagreement among experts increases, greater risks of exceeding a global temperature threshold also become more likely.

Note that this result is based entirely on the distribution of exceedance probabilities: It is therefore unimportant whether experts differ with respect to their estimates of mean climate sensitivity, or their estimates of uncertainty surrounding that mean estimate, or both. The only constraint in our analysis of second-order uncertainty is, once again, that the mean exceedance probability (i.e., $\mu_{B}$ ) remains constant - it is only by keeping the mean constant that the effects of varying degrees of disagreement among experts can be modeled.

In the supplementary material (Section S4), we apply the immediately preceding analysis to the data from an expert-elicitation study (Morgan \& Keith, 1995). The data of Morgan and Keith are particularly suitable because they contain one outlying observation; namely, an expert who provided a very low but highly certain estimate of climate sensitivity. We show that removal of this outlier, which reduces ambiguity among experts, tends to reduce the likelihood of extreme risks of exceeding a global temperature threshold. Conversely, we show that if this outlying expert is replicated and replaces some other observations at random, the likelihood of exceeding a tolerable risk of excessive warming increases in most cases. For that analysis, both mean and variance of the distribution of exceedance probabilities was allowed to vary freely: We nonetheless found that greater second-order un- 


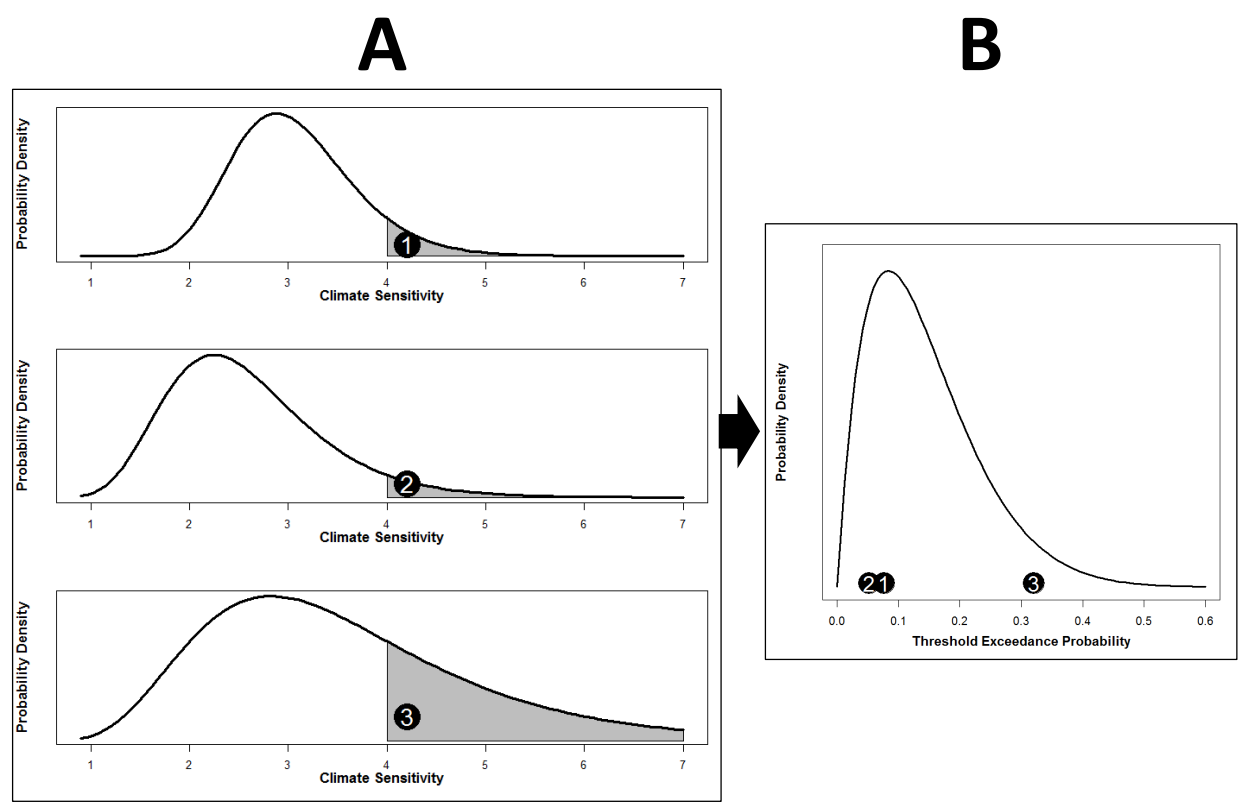

Fig. 4 A: Three hypothetical lognormal probability density functions (PDF) of climate sensitivity with different means and variances $\left(\mu_{G}=1.1,0.9\right.$, and 1.2 , respectively, from top to bottom; corresponding $\sigma_{G}=0.2,0.3$, and 0.4 ). Each distribution can be taken to represent the subjective PDF of an individual expert. The shaded areas, numbered 1 through 3 , identify the probabilities with which a temperature threshold $\left(T_{c}\right.$; in this case $4^{\circ} \mathrm{C}$ ) would be exceeded if $\mathrm{CO}_{2}$ levels doubled from preindustrial levels. B: Hypothetical Beta distribution (parameters $a=2 ; b=12$ ) that characterizes the exceedance probabilities (shaded areas in Panel $\mathrm{A} ; \pi_{i}$ 's) across individuals. Numbered points refer to shaded areas in Panel A. See text for more details.

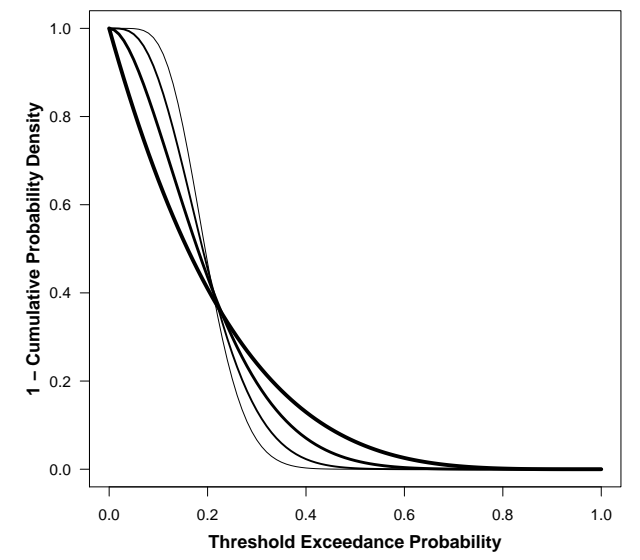

Fig. 5 Family of complementary cumulative distribution functions for Beta distributions of constant mean $\left(\mu_{B}=0.2\right)$ but differing variance. The standard deviations of the functions range from $\sigma_{B}=0.062$ for the lightest line, to $0.087,0.121$, and 0.163 in increasing order of line thickness. 
certainty entailed a greater likelihood of threshold exceedance in the majority of cases.

\subsection{Sea level rise and inundation risk}

Sea level rise (SLR) is one of the more costly consequences of climate change: Coping with SLR requires that in order to keep the risk of flooding constant, existing coastal structures must be relocated, raised, or protected by levees or dikes. To prevent an increase in the risk from flooding as sea levels rise, it is insufficient to focus on the projected mean SLR: Instead, an additional allowance for extreme events (e.g., storm surges) must be made.

The extra allowance for extreme events is a function of the uncertainty in the estimate of mean projected SLR, called uncertaintySLR from here on. That is, the total height of protective measures required to keep the risk from flooding constant is a function of the projected increase (call that $X$ ) as well as its uncertaintysLR (i.e., the standard deviation of $X$ ). Hunter (2012) computed the required magnitude of the constant-risk protective response to SLR. Risk here is measured in units of flooding events whose magnitude is assumed to remain constant. In line with recent recommendations (Katz et al., 2013), Hunter (2012) focused on modeling of extreme events. Specifically, Hunter used a Gumbel distribution, the simplest of the set of generalised extreme-value distributions, which is known to characterize sea-level extremes well (Van den Brink \& Können, 2011). The implications of Hunter (2012)'s analysis are shown in Figure 6.

The figure plots the total protective response that is necessary to cope with an



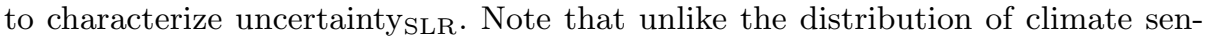
sitivity, the three modeled distributions for uncertaintysLR - uniform, Gaussian, and raised cosine - are all symmetrical. For the purposes of this illustration, SLR was expected to be $0.5 \mathrm{~m}$, represented by the horizontal dashed line. Accordingly, when there is no uncertainty SLR $_{\text {in }}$ the estimate, the total protective response can be equal to the expected SLR, namely $0.5 \mathrm{~m}$.

When uncertainty ${ }_{S L R}$ is non-zero, then irrespective of what assumptions are made about the distribution of SLR, the required protective response increases and deviates fairly rapidly and in an accelerating manner from the anticipated mean SLR. For example, if SLR is assumed to follow a Gaussian distribution, then if uncertainty $_{S L R}$ is around $0.36 \mathrm{~m}$, this raises the required protective response to the vicinity of $1 \mathrm{~m}$. That is, an expected SLR of $0.5 \mathrm{~m}$ requires that dikes and levees be raised by twice that amount in order to keep the risk of flooding constant in light

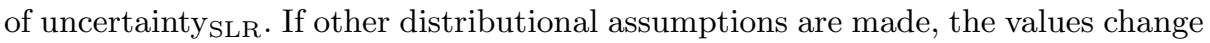
slightly but the in-principle conclusion remains the same: Greater uncertaintysLR translates into a greater required protective response. Note that the value of $0.36 \mathrm{~m}$ was not chosen arbitrarily but represents a current estimate of the uncertaintysLR in SLR projections, derived by Hunter (2012) based on the projections summarized by Nicholls et al. (2011). Likewise, the expectation of $0.5 \mathrm{~m}$ global mean SLR is consonant with IPCC projections for century's end on the current emissions trajectory (Meehl et al., 2007), although that estimate is now considered a lower bound on likely SLR (e.g., Nicholls et al., 2011). 




Fig. 6 The effects of uncertainty SLR $_{\text {in }}$ future sea level rise (SLR) on the protective response required to keep the risk from flooding constant. Protective response is expressed in $m$ (e.g., of raising dikes, levees, or buildings) and uncertainty SLR $_{\text {is }}$ expressed as the standard deviation $(\sigma)$ of the expected SLR. For this illustration, sea level is expected to rise by $0.5 \mathrm{~m}$ (dotted horizontal line). The three lines represent different distributional assumptions about future SLR. Figure produced from the equations provided by Hunter (2012).

Two points are worth underscoring in connection with Figure 6: First, the increase in the required protective response results from greater uncertaintysLR only - the mean expected SLR is constant for all data points in the figure. Second, the effects of uncertaintysLR derive directly from the mathematical properties of extreme values (for details, see Hunter, 2012), without any auxiliary assumptions such as the convexity of the economic damage function, and are therefore in little doubt at least at an ordinal level.

\section{Conclusions: Uncertainty and Unabated Emissions}

\subsection{Potential objections and limitations}

Unlike related precedents (e.g., Tomassini et al., 2010; Webster et al., 2003; Weitzman, 2009, 2011), our analysis ignored sources of uncertainty other than that associated with climate sensitivity or SLR. There are several other sources of uncertainty, for example potential amplifying loops in the carbon cycle itself: Because warming can accelerate respiration, soil may turn from a carbon sink to a carbon source (Cox et al., 2004), thereby shortening the time until $\mathrm{CO}_{2}$ has doubled. The considerable uncertainty about the global policy response likewise affects the time of $\mathrm{CO}_{2}$ doubling. One may therefore question whether our analysis extends to those other sources of uncertainty.

Webster et al. (2003) differentiated between climate and emissions uncertainty, suggesting that either on its own is just over half the uncertainty of both combined. Specifically, the standard deviation of expected temperature increases by 2100 was $0.69^{\circ} \mathrm{C}$ for climate uncertainty alone, $0.76^{\circ} \mathrm{C}$ for emissions uncertainty, and $1.18^{\circ} \mathrm{C}$ for both combined. Emissions uncertainty thus adds to the uncertaintyeCs we 
have considered here, implying that this additional source of uncertainty merely amplifies the impact of our analysis.

It must also be noted that our results were obtained under a conservative

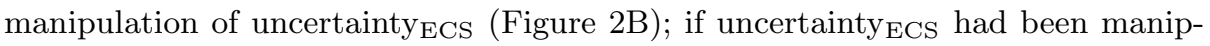
ulated while keeping the modal estimate for sensitivity constant (Figure 2A), our conclusions would have only been amplified because mean sensitivity would have increased together with uncertainty $y_{\mathrm{ECS}}$.

\subsection{Implications}

Our analyses of the role of uncertainty $\mathrm{ECS}_{\mathrm{S}}$ in relation to damage costs from climate change yielded a fairly clear conclusion: The greater the uncertainty about the evolution of the climate, the greater the expected cost of unmitigated global warming. When damages are considered at a macro-economic level, this relationship arises directly from the convexity of the damage function, an assumption shared by all extant economic models. When the consequences of climate change are considered with respect to sea level rise, the relationship between greater uncertaintysLR and greater adaptation costs emerges without making any assumptions about the cost function, simply from mathematical examination of the behavior of extreme values.

Our conclusion is unlikely to be affected by the discount rate because increasing uncertainty ${ }_{\mathrm{ECS}}$ likely also accelerates damages in addition to increasing their magnitude, thereby preventing any application of discounting. Similarly, because our analysis presents ordinal constraints (i.e., of the form "greater than") that do

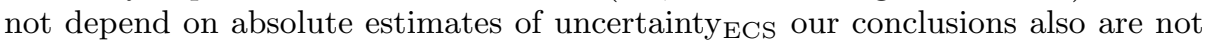
subject to cultural and personal risk perception variables. Our conclusion is also quite robust to "second-order" uncertainty, such as disagreement among experts about the climate-sensitivity distribution.

Our analysis permits at least one optimistic conclusion: The converse of our argument is that any reduction in uncertainty ${ }_{\mathrm{ECS}}$ arising from further research will translate into lesser expected damages from unmitigated climate change (cf. Webster et al., 2003). Recent findings that render extreme sensitivities less likely (Annan \& Hargreaves, 2011) are therefore encouraging from a risk management perspective.

At the outset, we identified two misconstruals of climatic uncertainty; viz. that surprises will likely be favorable, and that uncertainty prevents policy decisions from being scientifically informed. The analysis in this article has shown the first claim to be flawed: Any appeal to uncertainty about the evolution of the climate implies a stronger, rather than weaker, reason to be concerned about unmitigated climate change than in the absence of uncertainty. To complete the picture, we must confront the second misconstrual by analyzing whether uncertainty can prevent science from informing policy. This is addressed by the companion article (Lewandowsky et al., in press).

\section{References}

Ackerman, F., Heinzerling, L., \& Massey, R. (2005). Applying cost-benefit to past 
decisions: Was environmental protection ever a good idea? Administrative Law Review, 57, 155-192.

Aldred, J. (2009). The skeptical economist: Revealing the ethics inside economics. London: Earthscan.

Annan, J. D., \& Hargreaves, J. C. (2011). On the generation and interpretation of probabilistic estimates of climate sensitivity. Climatic Change, 104, 423-436.

Anthoff, D., Tol, R. S. J., \& Yohe, G. W. (2009). Discounting for climate change. Economics: The Open Access Open Assessment E-Journal, 3.

Bahn, O., Edwards, N. R., Knutti, R., \& Stocker, T. F. (2011). Energy policies avoiding a tipping point in the climate system. Energy Policy, 39, 334-348. doi: $10.1016 /$ j.enpol.2010.10.002

Bender, F. A.-M., Ekman, A. M., \& Rodhe, H. (2010). Response to the eruption of mount pinatubo in relation to climate sensitivity in the CMIP3 models. Climate dynamics, 35, 875-886.

Brewster, J. F., Graham, M. R., \& Mutch, A. C. (2005). Convexity, Jensen's inequality and benefits of noisy mechanical ventilation. Journal of the Royal Society Interface, 2, 393-396.

Cox, P. M., Betts, R. A., Collins, M., Harris, P. P., Huntingford, C., \& Jones, C. D. (2004). Amazonian forest dieback under climate-carbon cycle projections for the 21st century. Theoretical and Applied Climatology, 78, 137-156.

Feintuck, M. (2005). Precautionary maybe, but what's the principle? The precautionary principle, the regulation of risk, and the public domain. Journal of Law and Society, 32, 371-398. doi: 10.1111/j.1467-6478.2005.00329.x

Freudenburg, W. R., Gramling, R., \& Davidson, D. J. (2008). Scientific certainty argumentation methods (SCAMs): Science and the politics of doubt. Sociological Inquiry, 78, 2-38.

Freudenburg, W. R., \& Muselli, V. (2013). Reexamining climate change debates: Scientific disagreement or scientific certainty argumentation methods (SCAMs)? American Behavioral Scientist, 57, 777-795. doi: 10.1177/ 0002764212458274

Funtowicz, S. O., \& Ravetz, J. R. (1994). The worth of a songbird: ecological economics as a post-normal science. Ecological Economics, 10, 197-207.

Gardiner, S. (2006). A core precautionary principle. The Journal of Political Philosophy, 14, 33-60.

Garnaut, R. (2011). The Garnaut review 2011: Australia in the global response to climate change. Cambridge: Cambridge University Press.

Hansen, J., Russell, G., Lacis, A., Fung, I., Rind, D., \& Stone, P. (1985). Climate response times: Dependence on climate sensitivity and ocean mixing. Science, 229, 857-859.

Hartman, R. (1972). The effects of price and cost uncertainty on investment. Journal of Economic Theory, 5, 258-266.

Hegerl, G. C., Crowley, T. J., Hyde, W. T., \& Frame, D. J. (2006). Climate sensitivity constrained by temperature reconstructions over the past seven centuries. Nature, 440, 1029-1032.

Holmes, J., Lowe, J., Wolff, E., \& Srokosz, M. (2011). Rapid climate change: lessons from the recent geological past. Global and Planetary Change, 79, $157-162$.

Hunter, J. (2012). A simple technique for estimating an allowance for uncertain sea-level rise. Climatic Change, 113, 239-252. doi: 10.1007/s10584-011-0332 
$-1$

Intergovernmental Panel on Climate Change. (2005). Guidance notes for lead authors of the IPCC Fourth Assessment Report on addressing uncertainties. (Tech. Rep.).

Jensen, J. L. W. V. (1906). Sur les fonctions convexes et les inégalités entre les valeurs moyennes. Acta Mathematica, 30, 175-193.

John, S. (2010). In defence of bad science and irrational policies: an alternative account of the precautionary principle. Ethical Theory and Moral Practice, 13, 3-18. doi: 10.1007/s10677-009-9169-3

Kahan, D. M., Slovic, P., Braman, D., \& Gastil, J. (2006). Laws of fear: Beyond the precautionary principle. Harvard Law Review, 119, 1071-1109.

Katz, R. W., Craigmile, P. F., Guttorp, P., Haran, M., Sansó, B., \& Stein, M. L. (2013). Uncertainty analysis in climate change assessments. Nature Climate Change, 3, 769-771.

Knutti, R., \& Hegerl, G. C. (2008). The equilibrium sensitivity of the Earth's temperature to radiation changes. Nature Geoscience, 1, 735-743.

Lewandowsky, S., Oreskes, N., Newell, B. R., Smithson, M., \& Risbey, J. S. (2013). Uncertainty as a barrier to mitigation: Historical and psychological factors. Manuscript in preparation.

Lewandowsky, S., Risbey, J. S., Smithson, M., \& Newell, B. R. (in press). Scientific uncertainty and climate change: Part II. Uncertainty and mitigation. Climatic Change.

Li, M., Vietri, J., Galvani, A. P., \& Chapman, G. B. (2010). How do people value life? Psychological Science, 21, 163-167. doi: 10.1177/0956797609357707

Meehl, G. A., Stocker, T. F., Collins, W. D., Friedlingstein, P., Gaye, A. T., Gregory, J. M., ... Zhao, Z.-C. (2007). Global climate projections. In S. Solomon et al. (Eds.), Climate change 200\%: The physical science basis. contribution of working group I to the Fourth Assessment Report of the Intergovernmental Panel on Climate Change (pp. 748-845). Cambridge: Cambridge University Press.

Michaels, D. (2008). Doubt is their product: How industry's assault on science threatens your health. New York: Oxford University Press.

Morgan, M. G., \& Keith, D. W. (1995). Subjective judgments by climate experts. Environmental Science 8 Technology, 29, 468A-476A.

Narita, D. (2012). Managing uncertainties: The making of the IPCCs Special Report on Carbon Dioxide Capture and Storage. Public Understanding of Science, 21, 84-100.

Nicholls, R. J., Marinova, N., Lowe, J. A., Brown, S., Vellinga, P., De Gusmão, D., ... Tol, R. S. J. (2011). Sea-level rise and its possible impacts given a 'beyond $4^{\circ} \mathrm{C}$ ' world in the twenty-first century. Philosophical Transactions of the Royal Society (A), 369, 161-181.

Nolt, J. (2011). How harmful are the average American's greenhouse gas emissions? Ethics, Policy \& Environment, 14, 3-10.

Nordhaus, W. D. (2010). Economic aspects of global warming in a postCopenhagen environment. Proceedings of the National Academy of Science, $107,11721-11726$.

Nordhaus, W. D. (2011). The economics of tail events with an application to climate change. Review of Environmental Economics and Policy, 5, 240-257.

Padilla, L. E., Vallis, G. K., \& Rowley, C. W. (2011). Probabilistic estimates of transient climate sensitivity subject to uncertainty in forcing and natural 
variability. Journal of Climate, 24, 5521-5537. doi: 10.1175/2011JCL13989.1

Peterson, M. (2006). The precautionary principle is incoherent. Risk Analysis, 26 , 595-601. doi: 10.1111/j.1539-6924.2006.00781.x

Raupach, M. R., Canadell, J. G., Ciais, P., Friedlingstein, P., Rayner, P. J., \& Trudinger, C. M. (2011). The relationship between peak warming and cumulative $\mathrm{CO} 2$ emissions, and its use to quantify vulnerabilities in the carbonclimate-human system. Tellus, 63B, 145-164.

Ressurreição, A., Gibbons, J., Dentinho, T. P., Kaiser, M., Santos, R. S., \& Edwards-Jones, G. (2011). Economic valuation of species loss in the open sea. Ecological Economics, 70, 729-739.

Risbey, J. S. (2006). Some dangers of 'dangerous' climate change. Climate Policy, $6,527-536$.

Rive, N., \& Myhre, G. (2012). Communicating the probabilities of extreme surface temperature outcomes. Atmospheric and Climate Sciences, 2, 538-545.

Roe, G. H., \& Armour, K. C. (2011). How sensitive is climate sensitivity? Geophysical Research Letters, 38, L14708. doi: 10.1029/2011GL047913

Roe, G. H., \& Baker, M. B. (2007). Why is climate sensitivity so unpredictable? Science, 318, 629-632.

Roe, G. H., \& Baker, M. B. (2011). Comment on "Another look at climate sensitivity" by Zaliapin and Ghil (2010). Nonlinear Processes in Geophysics, $18,125-127$.

Ross, A., Matthews, H. D., Schmittner, A., \& Kothavala, Z. (2012). Assessing the effects of ocean diffusivity and climate sensitivity on the rate of global climate change. Tellus B, 64, 17733.

Sarewitz, D. (2004). How science makes environmental controversies worse. Environment Science \& Policy, 7, 385-403.

Schneider, S. H. (2002). Can we estimate the likelihood of climatic changes at 2100? Climatic Change, 52, 441-451.

Sherwood, S. C., \& Huber, M. (2010). An adaptability limit to climate change due to heat stress. Proceedings of the National Academy of Science, 107, 95529555.

Slovic, P. (1999). Trust, emotion, sex, politics, and science: Surveying the riskassessment battlefield. Risk Analysis, 19, 689-701.

Smallwood, P. D. (1996). An introduction to risk sensitivity: The use of Jensen's Inequality to clarify evolutionary arguments of adaptation and constraint. American Zoologist, 36, 392-401.

Smithson, M. (1999). Conflict aversion: Preference for ambiguity vs conflict in sources and evidence. Organizational Behavior and Human Decision Processes, $79,179-198$.

Stern, N. (2007). The economics of climate change: The Stern review. Cambridge: Cambridge University Press.

Sterner, T., \& Persson, U. M. (2008). An even Sterner review: Introducing relative prices into the discounting debate. Review of Environmental Economics and Policy, 2, 61-76.

Tol, R. S. J. (2009). The economic effects of climate change. Journal of Economic Perspectives, 23, 29-51.

Tol, R. S. J. (2011). The social cost of carbon. Annual Review of Resource Economics, 3, 419-443. 
Tomassini, L., Knutti, R., Plattner, G.-K., van Vuuren, D. P., Stocker, T. F., Howarth, R. B., \& Borsuk, M. E. (2010). Uncertainty and risk in climate projections for the 21st century: comparing mitigation to non-intervention scenarios. Climatic Change, 103, 399-422. doi: 10.1007/s10584.009.9763.3

Van der Sluijs, J. P. (2005). Uncertainty as a monster in the science-policy interface: four coping strategies. Water Science \&6 Technology, 52(6), 87-92.

Van den Brink, H. W., \& Können, G. P. (2011). Estimating 10000-year return values from short time series. International Journal of Climatology, 31, 115126.

Vlek, C. (2010a). Judicious management of uncertain risks: I. Developments and criticisms of risk analysis and precautionary reasoning. Journal of Risk Research, 13, 517-543.

Vlek, C. (2010b). Judicious management of uncertain risks: II. Simple rules and more intricate models for precautionary decision-making. Journal of Risk Research, 13, 545-569. doi: 10.1080/13669871003629903

Walley, P. (1991). Statistical reasoning with imprecise probabilities. London: Chapman and Hall.

Webster, M., Forest, C., Reilly, J., Babiker, M., Kicklighter, D., Mayer, M., .. . Wang, C. (2003). Uncertainty analysis of climate change and policy response. Climatic Change, 61, 295-320. doi: 10.1023/B:CLIM.0000004564.09961.9f

Weitzman, M. L. (2009). On modeling and interpreting the economics of catastrophic climate change. Review of Economics and Statistics, 91, 1-19.

Weitzman, M. L. (2010a). How should the distant future be discounted when discount rates are uncertain? Economic Letters, 107, 350-353.

Weitzman, M. L. (2010b). What is the "damages function" for global warming and what difference might it make? Climate Change Economics, 1, 57-69.

Weitzman, M. L. (2011). Fat-tailed uncertainty in the economics of catastrophic climate change. Review of Environmental Economics and Policy, 5, 275-292.

Winton, M., Adcroft, A., Griffies, S. M., Hallberg, R. W., Horowitz, L. W., \& Stouffer, R. J. (2013). Influence of ocean and atmosphere components on simulated climate sensitivities. Journal of Climate, 26 (1), 231-245.

Wouter Botzen, W., \& van den Bergh, J. C. (2012). How sensitive is Nordhaus to Weitzman? climate policy in DICE with an alternative damage function. Economics Letters, 117(1), 372-374.

Zeebe, R. E., Zachos, J. C., \& Dickens, G. R. (2009). Carbon dioxide forcing alone insufficient to explain Palaeocene-Eocene Thermal Maximum warming. $\mathrm{Na}$ ture Geoscience, 2(8), 576-580.

Acknowledgements Preparation of this paper was facilitated by a Discovery Grant and a Discovery Outstanding Researcher Award from the Australian Research Council, and a Wolfson Research Merit Award from the Royal Society, to the first author, by a Future Fellowship from the Australian Research Council to Ben Newell, and funding from the Australian Research Council Centre of Excellence in Climate Systems Science. The work was also supported by a Linkage Grant from the Australian Research Council, by the National Climate Change Adaptation Research Facility, and the CSIRO Climate Adaptation Flagship. We thank four reviewers for their incisive critique and helpful comments. Correspondence to the first author at the School of Experimental Psychology, University of Bristol, 12A Priory Road, Bristol BS8 1TU, United Kingdom (stephan.lewandowsky@bristol.ac.uk). Personal web page: http://www.cogsciwa.com. 